\title{
Morphine Induces Synchronous Oscillatory Discharges in the Rat Locus Coeruleus
}

\author{
Hong Zhu' ${ }^{1}$ and Wu Zhou ${ }^{2}$ \\ Departments of ${ }^{1}$ Pharmacology and Toxicology and ${ }^{2}$ Surgery/Otolaryngology, Neurology and Anatomy, University of \\ Mississippi Medical Center, Jackson, Mississippi 39216
}

\begin{abstract}
The noradrenergic locus coeruleus (LC) plays a role in opioid dependence and withdrawal. In the present study, using a multiple-electrode recording technique that allowed several LC neurons to be recorded simultaneously over long time periods, LC neuronal activities were recorded before and after intracerebroventricular injection of morphine (26 nmol) under halothane anesthesia. We found that morphine did not simply decrease firing rates of LC neurons, as reported in earlier studies, but that it induced persistent oscillatory discharges in 49\% (87 of 178) of the LC neurons recorded. Cross-correlation analysis revealed that almost all LC neurons (86 of 87 ) that exhibited oscillatory discharges were synchronized with at least one other neuron. When stated in terms of simultaneously recorded neuron pairs, 59\% (292 of 492) of the oscillatory neuron pairs discharged synchronously. The morphine-induced synchronous oscillation began at $\sim 10$ min after morphine injection,
\end{abstract}

reached its peak in $\sim 20-30$ min, persisted throughout the recording periods (up to 110 min after morphine injection, the longest recording time), and were reversed by an opioid receptor antagonist naltrexone. These data suggest that although the overall firing rate of LC neurons was reduced by morphine, the morphine-induced synchronous oscillatory activity may summate temporally and spatially at LC axon terminals and facilitate release of noradrenaline. Noradrenaline is an important neuromodulator and has been shown to induce and facilitate synaptic plasticity at LC target sites. We propose that the morphine-induced long-lasting synchronous oscillatory activity in the LC may be a neuronal signal that could induce synaptic plasticity leading to opioid addiction.

Key words: locus coeruleus; morphine; synchronous oscillation; multiple-electrode recording; noradrenaline; synaptic plasticity
The brain locus coeruleus (LC) is the largest cluster of noradrenergic neurons in the brain and projects broadly throughout the CNS (for review, see Foote et al., 1983). The LC is enriched with opioid receptors (Temple and Zukin, 1987) and plays a role in several effects of opioids, such as opioid dependence and withdrawal (for review, see Nestler et al., 1994; Nestler and Aghajanian, 1997; Zhu et al., 1998).

Systemic or intracoerulear administration of opioids, such as morphine, has been shown to have an inhibitory action on spontaneous LC neuronal activity (Korf et al., 1974; Bird and Kuhar, 1977; Aghajanian, 1978; Valentino and Wehby, 1988). These earlier electrophysiological studies have focused on the effects of opioids on the activity of individual LC neurons. Several recent studies indicate that temporal relationships among the activities of LC neurons can also be modulated under certain circumstances. For example, synchronous membrane potential in the LC was found in explant tissue cultures or brain slices prepared from neonatal (Finlayson and Marshall, 1988; Christie et al., 1989; Christie and Jelinek, 1993) and adult rats (Travagli et al., 1995; Ishimatsu and Williams, 1996). Synchronized LC firing was also observed in monkeys performing visual discrimination tasks (Usher et al., 1999). The synchronous activities in the LC have

\footnotetext{
Received June 5, 2001; revised July 25, 2001; accepted Aug. 10, 2001.

We thank Drs. I. K. Ho and W. Turner for their support, Drs. K. L. Simpson and C.-S. Lin for their help in the preliminary phase of the study, Jerome Allison for his technical support, and Drs. W. M. King, J. P. Shaffery, and T. P. Ma for their comments on this manuscript.

Correspondence should be addressed to Dr. Hong Zhu, Department of Pharmacology and Toxicology, University of Mississippi Medical Center, 2500 North State Street, Jackson, MS 39216. E-mail: zhu@vor.umsmed.edu.

Copyright (C) 2001 Society for Neuroscience $\quad 0270-6474 / 01 / 210001-05 \$ 15.00 / 0$
}

been proposed to have important implications in development (trophic role) (Christie et al., 1989) and regulation of cognitive performance (Usher et al., 1999). However, the effect of opioids on the temporal correlation between LC neurons has not been studied systemically in whole animals. In the present study, using a multiple-electrode recording technique that allowed several LC neurons to be recorded simultaneously over long time periods, we examined the effect of morphine, a classic opioid drug, on the temporal correlation between LC neurons.

\section{MATERIALS AND METHODS}

Surgery. All procedures were approved by the Institutional Animal Care and Use Committee at University of Mississippi Medical Center. Adult male Sprague Dawley rats $(250-350 \mathrm{gm})$ were used in this study. A bundle of eight microwires (40 $\mu \mathrm{m} /$ wire; NB Labs, Dennison, TX) was stereotaxically implanted into the LC under sodium pentobarbital (50 $\mathrm{mg} / \mathrm{kg}$, i.p.) anesthesia. A 21 gauge guide cannula was implanted into the lateral cerebral ventricle for drug injection. The microwire bundle and guide cannula were secured in place with four stainless steel screws

This article is published in The Journal of Neuroscience, Rapid Communications Section, which publishes brief, peerreviewed papers online, not in print. Rapid Communications are posted online approximately one month earlier than they would appear if printed. They are listed in the Table of Contents of the next open issue of JNeurosci. Cite this article as: JNeurosci, 2001, 21:RC179 (1-5). The publication date is the date of posting online at www.jneurosci.org.

http://www.jneurosci.org/cgi/content/full/5759 
trepanned through the skull and adhered with dental acrylic. Rats were given at least 1 week to recover after the surgery before recording.

Electrophysiological recordings. LC neuronal activities were recorded before and after intracerebroventricular injection of morphine solution (26 nmol, $5 \mu \mathrm{l}$, in saline) under halothane anesthesia $(1.25 \%$, mixed with oxygen). Body temperature was maintained at $37^{\circ} \mathrm{C}$ with a heating pad. Online isolation and discrimination of neuronal activity was accomplished using a commercial multichannel neuronal acquisition processor (MNAP) system (Plexon, Dallas, TX) that allows one to monitor groups (up to four neurons per wire) of neurons simultaneously. Identifying different neurons on a single wire was accomplished by real-time discrimination of individual waveforms using template analysis procedures provided by the MNAP system. To ensure that neurons recorded by different wires were distinct, we compared the shape of their waveforms, firing rates, and patterns (e.g., interspike interval histograms) before further analysis. LC neurons were identified using previously established criteria, i.e., low spontaneous firing rates, responses to noxious stimuli, and changes in firing rates in response to morphine (Korf et al., 1974; Bird and Kuhar, 1977; Aghajanian, 1978; Valentino and Wehby, 1988). At the end of recording, currents $(30 \mu \mathrm{A}, 15 \mathrm{sec})$ were passed through the microwires to create lesions that were verified histologically.

Data analysis. Mean firing rates, autocorrelograms, and crosscorrelograms were analyzed using Nex (Nex Technologies, Lexington, MA) and Matlab (Mathworks, Natick, MA) software programs. The degree of oscillation was quantified by an oscillatory index, which was computed as the ratio of the amplitude of the first satellite peak to the offset of the autocorrelogram (König, 1994). The strength of synchrony was quantified by a synchrony index, which was computed as the ratio of the amplitude of the central peak to the offset of the cross-correlogram (König, 1994). The mean firing rates, oscillatory indexes, and synchrony indexes before and after morphine administration were compared using paired $t$ test. Data are presented as mean \pm SEM.

\section{RESULTS}

A total of 408 neurons were recorded from 19 adult rats before and after intracerebroventricular injection of morphine. One hundred and seventy-eight neurons in 10 rats were identified as LC neurons using established criteria (see Materials and Methods). The mean spontaneous firing rates of the LC neurons decreased $48 \%$, from $4.0 \pm 0.5$ spikes $/ \mathrm{sec}$ to $2.1 \pm 0.3 \mathrm{spikes} / \mathrm{sec}$ ( $n=178 ; p<0.0001$; paired $t$ test) $10 \mathrm{~min}$ after intracerebroventricular injection of morphine $(26 \mathrm{nmol}, 5 \mu \mathrm{l})$. We found that a subpopulation of the LC neurons (87 of 178; 49\%) not only exhibited decreases in their mean firing rates, but also exhibited repeated bursts of discharge activity. The discharge patterns of two representative LC neurons recorded simultaneously are shown in Figure 1. In addition to a decreased firing rate, the two LC neurons exhibited bursts of action potentials 20 min after morphine injection (Fig. 1a). At higher temporal resolution, we observed that the bursts occurred regularly ( $\sim 90 \mathrm{sec} / \mathrm{cycle}$ ) (Fig. $1 b, c$, right panels). The regularity was confirmed by their autocorrelograms, which showed distinctive satellite peaks after morphine injection (Fig. 1d, filled histogram). The degree of regularity was quantified by an oscillatory index. Twenty minutes after morphine injection, $23.3 \pm 2.4 \%(n=87)$ of the LC neuronal activity was deemed oscillatory, compared with $0.3 \pm 0.1 \%$ of the activity ( $n=87 ; p<0.0001$; paired $t$ test) before morphine. The length of the average cycle of the morphine-induced oscillation was $109.3 \pm 3.2 \sec (n=87)$.

An earlier study showed similar burst discharges in the LC after morphine injection in an awake monkey (Aston-Jones et al., 1992). However, because single electrodes were used in that study, it was unable to determine the temporal correlations between LC neurons. In the present study, using the multiplechannel recording technique, we were able to examine if the morphine-induced bursts in LC neurons were temporally correlated. Figure 1, $b$ and $c$ (right panels), shows that the morphineinduced oscillatory bursts were temporally correlated between the two LC neurons that were recorded simultaneously, as indicated by the dotted vertical lines. This apparent synchrony was confirmed statistically by computing the cross-correlation of the discharge activity of the pair of LC neurons. Their crosscorrelograms showed a significant central peak (centered at 0.1 msec time lag) (Fig. 1e, filled histogram) after morphine, indicating that this pair of LC neurons discharged synchronously.

In individual rats, several LC neurons (2-21 neurons per rat) that exhibited oscillatory discharges were recorded simultaneously. The degree of synchrony between possible pairs of oscillatory LC neurons in each rat was analyzed. We analyzed a total of 492 neuron pairs from nine rats. Fifty-nine percent of the LC neuron pairs (292 of 492) exhibited synchronous oscillation after morphine. When stated in terms of neurons rather than pairs, however, almost all neurons (86 of 87) were involved in synchronous oscillation with at least one other neuron after morphine. It is not surprising that the percentage of neurons involved in synchrony is larger than that of pairs showing synchrony, because oscillatory LC neurons recorded in individual rats did not always discharge synchronously as a single group. In six rats, the oscillatory LC neurons formed a single synchronous group in each rat. In the other three rats, however, the oscillatory LC neurons formed two or three subgroups in each rat, and synchrony was only present between neurons within the same subgroup. The strength of the synchrony between a pair of LC neurons was quantified by a synchrony index. Twenty minutes after morphine injection, $31.5 \pm 2.3 \%$ ( $n=292$ pairs) of the LC neuronal activity was synchronous, compared with $6.4 \pm 0.6 \%$ ( $n=292 ; p<0.0001$; paired $t$ test) before morphine.

To examine the time course of the morphine-induced synchronized oscillation, the oscillatory indexes of the $87 \mathrm{LC}$ neurons and the synchrony indexes of the 292 LC neuron pairs were computed and averaged every $10 \mathrm{~min}, 30 \mathrm{~min}$ before morphine injection and 110 min afterward (Fig. 2a). The morphine-induced oscillation began at $\sim 10 \mathrm{~min}$ after morphine injection, reached its peak in $\sim 20-30 \mathrm{~min}$, and persisted throughout the recording periods (up to $110 \mathrm{~min}$ after morphine injection, the longest recording time). The morphine-induced synchrony showed a strikingly similar time course. Moreover, the oscillatory indexes were highly correlated to the synchrony indexes (Fig. $2 b)\left(r^{2}=0.87\right)$. Because there was a strong correlation between the time course of synchrony and that of oscillation in LC neurons (Fig. 2), they may share a common mechanism (Singer, 1993).

In addition to the $87 \mathrm{LC}$ neurons that exhibited both decreases in firing rates and synchronous oscillations, another subpopulation of LC neurons (91 of 178, 51\%) exhibited sustained decreases in firing rates, but neither oscillatory discharges nor synchrony after morphine injection. These two subpopulations of LC neurons that responded to morphine in the two different ways were found in almost every individual rats (9 of 10). However, these two subpopulations of LC neurons were indistinguishable in terms of mean firing rates before $(4.1 \pm 0.5 \mathrm{spikes} / \mathrm{sec}, n=87 \mathrm{vs}$ $3.9 \pm 0.5$ spikes/sec, $n=91 ; p>0.5 ; t$ test) and 10 min after morphine $(2.2 \pm 0.3$ spikes $/ \mathrm{sec}, n=87$, vs $2.0 \pm 0.3$ spikes $/ \mathrm{sec}$, $n=91 ; p>0.7 ; t$ test) (Fig. 3). Their mean firing rates did not recover $110 \mathrm{~min}$ after morphine.

Effect of systemic administration of morphine on the firing pattern of LC neurons was also examined. Among the 27 LC neurons recorded after intravenous administration of morphine $(1.25 \mathrm{mg} / \mathrm{kg}), 17$ of them exhibited both decreases in mean firing rates and synchronous oscillatory discharges. Their oscillatory indexes were $23.6 \pm 2.6 \%$ after morphine compared with $2.5 \pm$ 


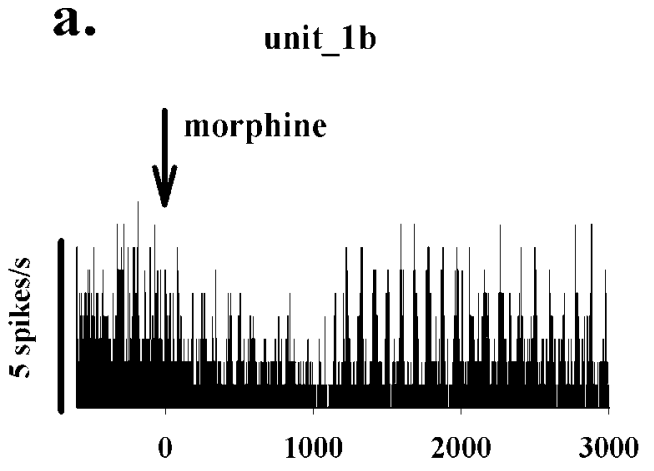

b. unit_8a: before morphine

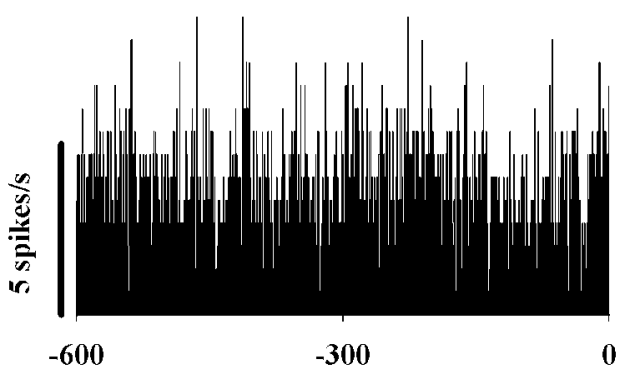

C. unit_1b: before morphine

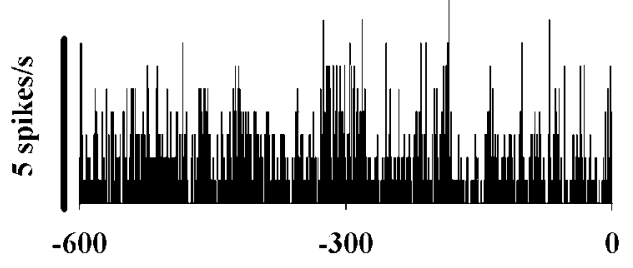

d. Autocorrelogram :unit_1b

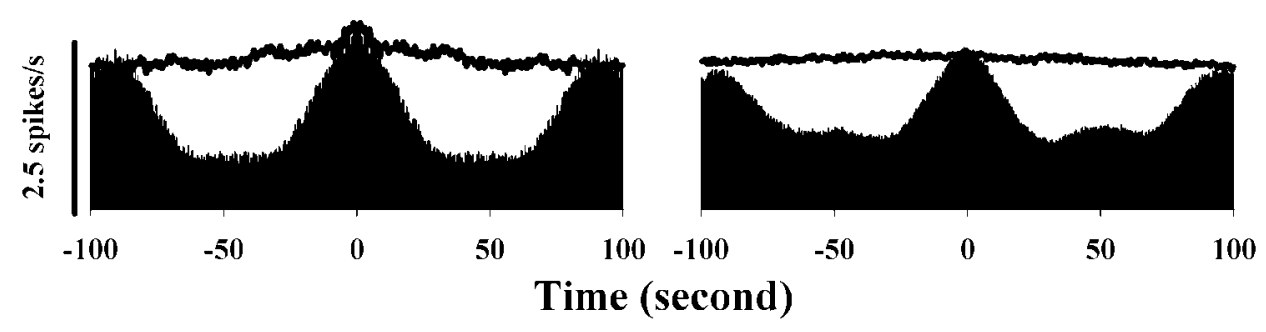

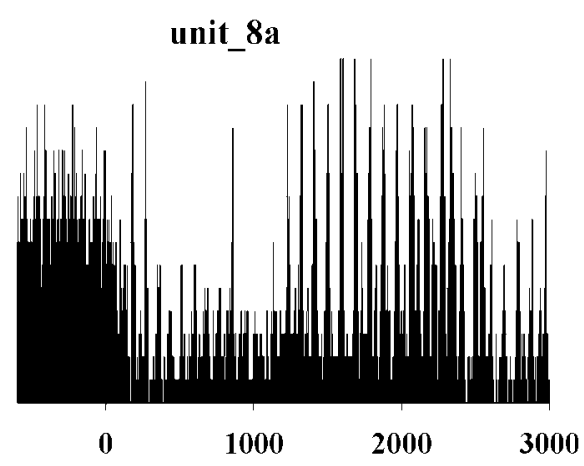
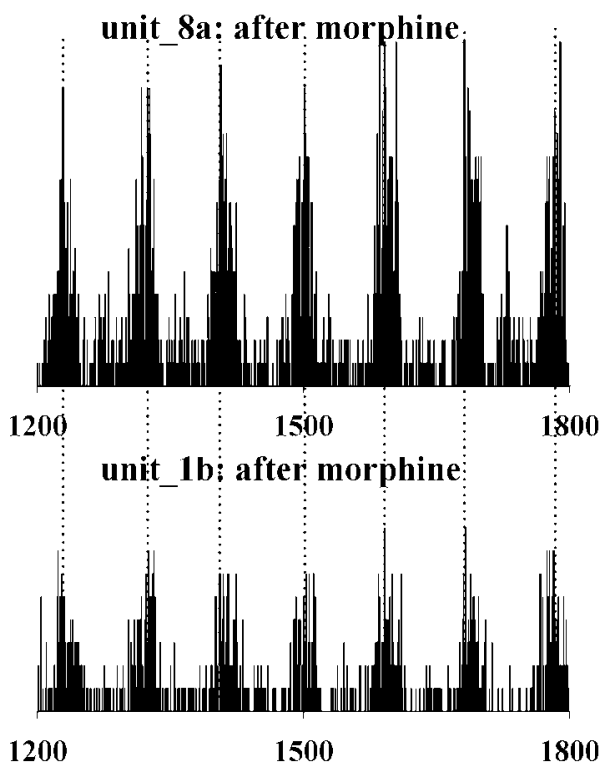

1800

Figure 1. Effects of morphine on the firing patterns of two simultaneously recorded LC neurons (unit $1 \mathrm{~b}$ and unit $8 \mathrm{a}$ ). $a$, Histograms of spontaneous firing rate of the two LC neurons before and after intracerebroventricular morphine injection $(26 \mathrm{nmol}$, at 0 time). The bin size is $1 \mathrm{sec} . b, c$, The discharges of the same two LC neurons are shown at higher temporal resolution (10 min epochs, before and after morphine). The dotted lines show that the two LC neurons oscillated synchronously after morphine. $d$, Autocorrelograms of unit_1b before (coarse line) versus $20 \mathrm{~min}$ after (filled histograms) morphine. Note the lack of satellite peaks before morphine versus large satellite peaks after morphine (oscillatory index of unit 1b: 0 vs $48.2 \%$ ). $e$, Crosscorrelograms between the two LC neurons before (coarse line) versus $20 \mathrm{~min}$ after ( filled histograms) morphine. Note a large central peak indicating synchronous activity after morphine injection. The synchronous index of this pair of LC neurons was $26.0 \%$ after morphine compared with $1.3 \%$ before morphine.

$0.2 \%$ before morphine ( $p<0.001$; paired $t$ test; $n=17$ ). Their synchronous indexes were $35.9 \pm 2.7 \%$ after morphine compared with $1.3 \pm 0.04 \%$ before morphine $(p<0.001$; paired $t$ test; $n=$ 136 pairs). The other $10 \mathrm{LC}$ neurons showed decreases in firing rates but showed neither oscillatory discharges nor synchrony after systemic administration of morphine. Thus, the systemic administration of morphine produced the same actions as did the intracerebroventricular administration of morphine. These data suggested that the synchronous oscillation observed after intracerebroventricular administration of morphine was not attributable to an unexpected effect of morphine that was injected by the intracerebroventricular route. Furthermore, the synchronous oscillation observed after morphine administration was reversed by an opioid antagonist naltrexone $(0.1 \mathrm{mg} / \mathrm{kg}$, i.v. $)$, indicating that the morphine-induced synchronous oscillation is opioid receptorspecific. The oscillatory indexes were $2.2 \pm 0.1 \%$ after naltrexone compared with $23.6 \pm 2.6 \%$ before naltrexone $(p<0.001$; paired $t$ test; $n=17)$. The synchronous indexes were $1.7 \pm 0.1 \%$ after naltrexone compared with $35.9 \pm 2.7 \%$ before naltrexone $(p<$ 0.001 ; paired $t$ test; $n=136$ pairs). In addition, LC neurons exhibited neither oscillation nor synchrony after saline injection (data not shown), suggesting that the observed synchronous oscillation was not attributable to halothane anesthesia or to mechanical disturbance of the tissue caused by the electrodes.

\section{DISCUSSION}

These results provide the first evidence that central or systemic administration of morphine does not simply decrease firing rates of LC neurons, as reported in earlier studies (Korf et al., 1974; Bird and Kuhar, 1977; Aghajanian, 1978; Valentino and Wehby, 1988), but that it induces long-lasting synchronous oscillatory discharges in a subpopulation of LC neurons. The morphineinduced synchronous oscillation can be reversed by an opioid receptor antagonist naltrexone. These results also suggest that the 

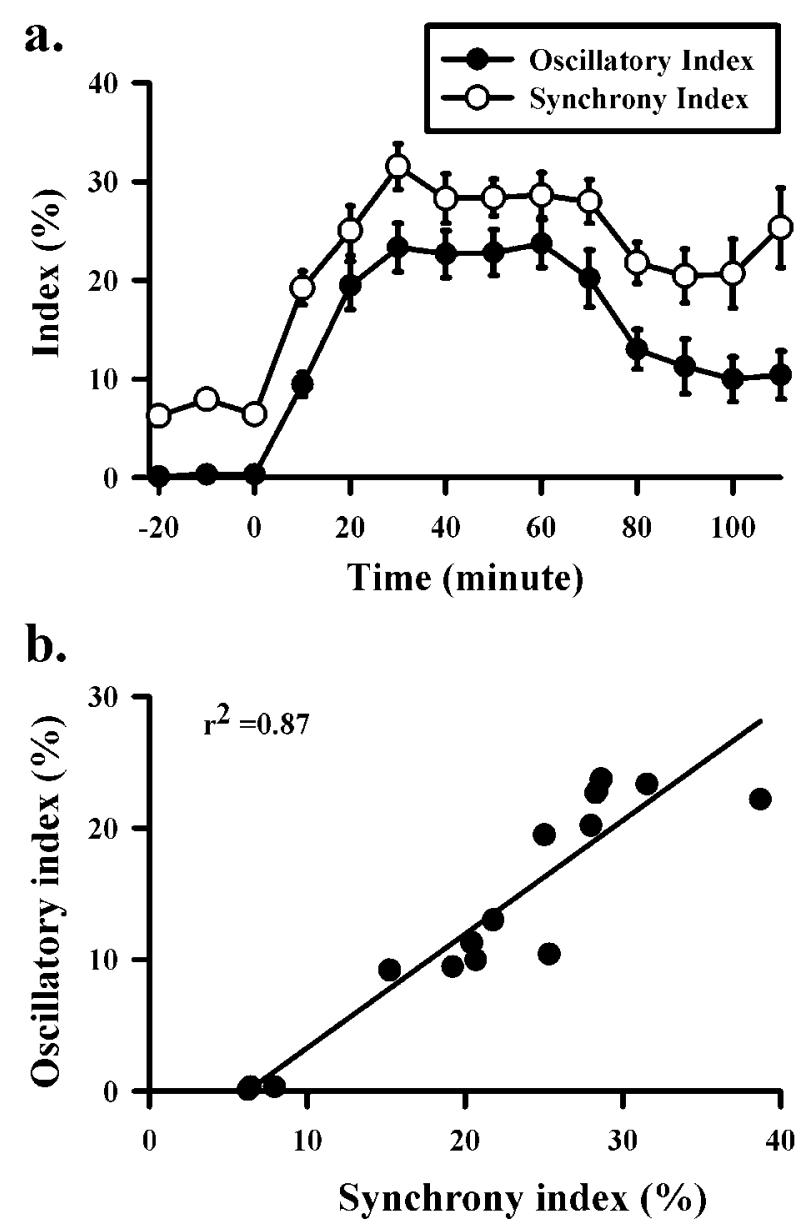

Figure 2. a, Time course of the oscillatory indexes of 87 LC neurons ( filled circle; mean \pm SEM) and synchronous indexes for 292 neuron pairs (open circle; mean \pm SEM) before and after intracerebroventricular injection of morphine. Morphine $(26 \mathrm{nmol}, 5 \mu \mathrm{l})$ was injected at time $0 . b$, Correlation between the synchronous indexes and the oscillatory indexes $\left(r^{2}=0.87 ; p<0.0001\right)$ after morphine.

responses of LC neurons to morphine were not homogeneous. There was another subpopulation of LC neurons that exhibited sustained decreases in firing rates, but did not exhibit synchronous oscillatory discharges after morphine injection.

There are two possible mechanisms underlying the morphineinduced synchronous oscillatory discharges in the LC. On one hand, electrotonic couplings in the LC, which have been shown in brain slices from neonatal rats (Christie et al., 1989; Christie and Jelinek, 1993) and adult rats (Travagli et al., 1995; Ishimatsu and Williams, 1996), could mediate the morphine-induced synchronous activity. On the other hand, central administration of morphine could induce synchronous oscillations in other brain areas that provide excitatory inputs to the LC, such as paragigantocellularis (Aston-Jones et al., 1986).

The morphine-induced synchronous oscillatory discharges in the LC may have a powerful influence on noradrenaline release in the widespread LC target areas. A microdialysis study has shown that noradrenaline output in the prefrontal cortex was inhibited initially by morphine injection, but progressively returned to baseline (Rossetti et al., 1993). This phenomenon cannot be simply explained by the changes in the mean firing rate of LC neurons that showed a sustained inhibition after morphine (Fig. 3) (Aston-Jones et al., 1992). However, the progressive

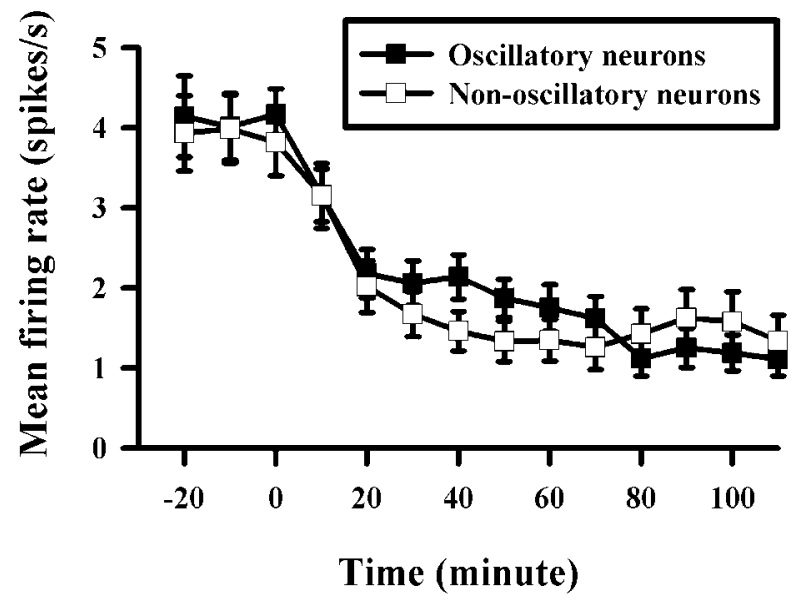

Figure 3. Effects of intracerebroventricular injection of morphine on the mean firing rates of LC neurons. Morphine $(26 \mathrm{nmol})$ was injected at time 0 . Data were presented as mean \pm SEM. Ninety-one LC neurons exhibited sustained decreases in spontaneous firing rates after morphine (nonoscillatory neurons, open squares). Eighty-seven LC neurons did not only exhibit decreases in firing rates, but also exhibited synchronous oscillatory discharges after morphine (oscillatory neurons, filled squares).

return of noradrenaline release could be explained by the morphine-induced synchronous oscillatory discharges reported here. Although the overall firing rate of LC neurons was reduced by morphine injection, as a result of temporal and spatial facilitation, the morphine-induced synchronous oscillatory activity could periodically induce increases in the release of noradrenaline in LC target areas.

Numerous studies have demonstrated that noradrenaline is a modulatory transmitter and can induce and facilitate long-lasting synaptic plasticity in LC target sites (Neuman and Harley, 1983; Lacaille and Harley, 1985; Winson and Dahl, 1985; Hopkins and Johnston, 1988; Huang and Kandel, 1996; Kirkwood et al., 1999; Huang et al., 2000) (for review, see Bailey et al., 2000). For example, a brief application of norepinephrine induced longlasting potentiation in the dentate gyrus in the absence of tetanic stimulation (Neuman and Harley, 1983; Lacaille and Harley, 1985; Winson and Dahl, 1985). In both the mossy fiber hippocampal pathway and cortico-amygdala pathway, $\beta$-adrenergic agonists facilitate the late phase of long-term potentiation (Hopkins and Johnston, 1988; Huang and Kandel, 1996; Huang et al., 2000). Synaptic plasticity has been hypothesized to be involved in mechanisms underlying the development of opioid addiction (for review, see Nestler, 2001, Williams et al., 2001). It has been demonstrated that chronic morphine treatment facilitates long-term potentiation of Schaffer collateral to CA1 synapses in hippocampus (Mansouri et al., 1999). Furthermore, blockades of NMDA receptors or nitric oxide have been shown to prevent the development of opioid dependence (Trujillo and Akil, 1991; Fundytus and Coderre, 1994; Majeed et al., 1994; Dambisya and Lee, 1996; Zhu and Ho, 1998). The opioid-induced synaptic plasticity may not only result from the local effect of opioid on neurons with opioid receptors, but also may result from the indirect effects of opioid. We propose that the morphine-induced persistent synchronous oscillatory discharges in the LC may periodically induce increases in noradrenaline release in the LC target areas, which may facilitate and/or initiate long-lasting synaptic plasticity leading to opioid addiction. 


\section{REFERENCES}

Aghajanian GK (1978) Tolerance of locus coeruleus neurons to morphine and suppression of withdrawal response by clonidine. Nature 276:186-188.

Aston-Jones G, Ennis M, Pieribone VA, Nickell WT, Shipley MT (1986) The brain nucleus locus coeruleus: restricted afferent control of a board afferent network. Science 234:734-737.

Aston-Jones G, Rajkowski J, Kubiak P, Akaoka H (1992) Acute morphine induces oscillatory discharge of noradrenergic locus coeruleus neurons in the waking monkey. Neurosci Lett 140:219-224.

Bailey CH, Giustetto M, Huang Y-Y, Hawkins RD, Kandel ER (2000) Is heterosynaptic modulation essential for stabilizing Hebbian plasticity and memory? Nat Rev Neurosci 1:11-20.

Bird SJ, Kuhar MJ (1977) Iontophoretic application of opiates to the locus coeruleus. Brain Res 122:523-533.

Christie MJ, Jelinek HF (1993) Dye-coupling among neurons of the rat locus coeruleus during postnatal development. Neuroscience 56:129-137.

Christie MJ, Williams JT, North RA (1989) Electrical coupling synchronizes subthreshold activity in locus coeruleus neurons in vitro from neonatal rats. J Neurosci 9:3584-3589.

Dambisya YM, Lee TL (1996) Role of nitric oxide in the induction and expression of morphine tolerance and dependence. $\mathrm{Br} \mathrm{J}$ Pharmacol 117:914-918

Finlayson PG, Marshall KC (1988) Synchronous burst of locus coeruleus neurons in tissue culture. Neuroscience 24:217-225.

Foote SL, Bloom FE, Aston-Jones G (1983) Nucleus locus coeruleus: new evidence of anatomical and physiological specificity. Physiol Rev 63:844-914.

Fundytus ME, Coderre TJ (1994) Effects of metabotropic as well as ionotropic (NMDA), glutamate receptors on morphine dependence. Br J Pharmacol 113:1215-1220.

Hopkins WF, Johnston D (1988) Noradrenergic enhancement of longterm potentiation at mossy fibers synapses in the hippocampus. J Neurophysiol 59:667-687.

Huang Y-Y, Kandel ER (1996) Modulation of both the early and the late phase of mossy fiber LTP by the activation of $\beta$-adrenergic receptors. Neuron 16:611-617.

Huang Y-Y, Martin KC, Kandel ER (2000) Both protein kinase A and mitogen-activated protein kinase are required in the amygdala for the macromolecular synthesis-dependent late phase of long-term potentiation. J Neurosci 20:6317-6325.

Ishimatsu M, Williams JT (1996) Synchronous activity in locus coeruleus results from dendritic interactions in pericoerulear regions. J Neurosci 16:5196-5204.

Kirkwood A, Rozas C, Kirkwood J, Perez F, Bear MF (1999) Modulation of long-term synaptic depression in visual cortex by acetylcholine and norepinephrine. J Neurosci 19:1599-1609.
König P (1994) A method for the quantification of synchrony and oscillatory properties of neuronal activity. J Neurosci Methods 54:31-37.

Korf J, Bunney BS, Aghajanian GK (1974) Noradrenergic neurons: morphine inhibition of spontaneous activity. Eur J Pharmacol 25:165-169.

Lacaille JC, Harley CW (1985) The action of norepinephrine in the dentate gyrus: beta-mediated facilitation of evoked potentials in vitro. Brain Res 358:210-220.

Majeed NH, Przewlocka B, Machelska H, Przewlocki R (1994) Inhibition of nitric oxide synthase attenuates the development of morphine tolerance and dependence in mice. Neuropharmacology 33:189-192.

Mansouri FA, Motamedi F, Fathollahi Y (1999) Chronic in vivo morphine administration facilitates primed-bursts-induced long-term potentiation of Schaffer collateral-CA1 synapses in hippocampal slices in vitro. Brain Res 815:419-423.

Nestler EJ (2001) Molecular basis of long-term plasticity underlying addiction. Nat Rev Neurosci 2:119-128.

Nestler EJ, Aghajanian GK (1997) Molecular and cellular basis of addiction. Science 278:58-63.

Nestler EJ, Altreja M, Aghajanian GK (1994) Molecular and cellular mechanisms of opiate action: studies in the rat locus coeruleus. Brain Res Bull 35:521-528.

Neuman RS, Harley CW (1983) Long-lasting potentiation of the dentate gyrus population spike by norepinephrine. Brain Res 273:162-165.

Rossetti ZL, Longu G, Mercuro G, Gessa GL (1993) Extraneuronal noradrenaline in the prefrontal cortex of morphine-dependent rats: tolerance and withdrawal mechanisms. Brain Res 609:316-320.

Singer W (1993) Synchronization of cortical activity and its putative role in information processing and learning. Annu Rev Physiol 55:349-374

Temple A, Zukin RS (1987) Neuroanatomical patterns of the $\mu, \delta$, and $\kappa$ opioid receptors of rat brain as determined by quantitative in vitro autoradiography. Proc Natl Acad Sci USA 84:4308-4312.

Travagli RA, Dunwiddie TV, Williams JT (1995) Opioid inhibition in locus coeruleus. J Neurophysiol 74:519-528.

Trujillo K, Akil H (1991) Inhibition of morphine tolerance and dependence by NMDA receptor antagonist MK801. Science 251:85-87.

Usher M, Cohen JD, Servan-Schreiber D, Rajkowski J, Aston-Jones G (1999) The role of locus coeruleus in the regulation of cognitive performance. Science 283:549-554.

Valentino RJ, Wehby RG (1988) Morphine effects on locus coeruleus neurons are dependent on the state of arousal and availability of external stimuli: studies in anesthetized and unanesthetized rats. J Pharmacol Exp Ther 244:1178-1186.

Williams JT, Christie MJ, Manzoni O (2001) Cellular and synaptic adaptations mediating opioid dependence. Physiol Rev 81:299-330.

Winson J, Dahl D (1985) Action of norepinephrine in the dentate gyrus. II. Iontophoretic studies. Exp Brain Res 59:497-506.

Zhu H, Ho IK (1998) NMDA-R1 antisense oligonucleotide attenuates withdrawal signs from morphine. Eur J Pharmacol 352:151-156.

Zhu H, Rockhold RW, Ho IK (1998) The role of glutamate in physical dependence on opioids. Jpn J Pharmacol 76:1-14. 